\title{
Multilingual Lexical Development : A case of Children of Indonesian Immigrants in
}

\section{Taiwan}

\section{Madina}

madina@unprimdn.ac.id

\begin{abstract}
The number of immigrants increases tremendously in Taiwan. These recent immigrants originate from a variety of ethnicities; some are Han Chinese from Mainland China, others are mainly from Southeast Asian countries, with Vietnamese as the largest group, followed by Indonesians, Thai, and then Philippinas. They brought with them their native languages and cultures (Chen,2010). Kuo (2008) found no difference in the lexical development of 2-6- year-old children of Vietnamese mothers and Taiwanese mothers, but children of Indonesian mothers were found to be slower (Kuo, 2015). This study aim to evaluate the the vocabulary size in Indonesian, Mandarin, and Taiwanese for children of Indonesian immigrants in Taiwan. The researcher investigated whether Indonesian mothers in Taiwan with limited Mandarin proficiency are able to provide the kind of input to facilitate their children's Indonesian, Mandarin and Taiwanese acquisition or not.This study used Peabody Picture Vocabulary Test to assess their lexical development in Indonesian and Mandarin. The Taiwanese language measure tool is an application developed by Kuo (2017). This study revealed that the vocabulary score of Indonesian score were found to be lower than Mandarin and Taiwanese score and Mandarin score higher than Taiwanese score. The factors related to children's lexical development were also investigated no correlation between Indonesian mother's with their children in Indonesian language, because most of them communicate with their limited Mandarin or Taiwanese. Finally, this research should help people to have a better understanding about the phenomenon of multilingual family, where multilingual has become common in the society.
\end{abstract}

Keyword : Multilingualism, lexical development, immigrant children, vocabulary size 


\section{CHAPTER 1 \\ INTRODUCTION}

\section{Background}

Many countries enjoy long histories of bi- or multilingualism; while, many traditionally monolingual countries are experiencing increasing bilingualism due to immigration (August and Shanahan, 2006). Most of the researchers have focused on examining the type of immigration that typically involves entire families migrating from one country to another, in which the individuals within the families all adapt together to a new culture, language, value system, and physical environment (Chian \& Chen, 2011).

Many mainstream families, in general, and immigrant families, in particular express strong interest in raising their children bilingually for a variety of reasons. Many families believe that children who are bilingual will be able to communicate with parents and other family members, they will benefit from the cognitive, academic, and social advantages of being bilinguals; and they will also have improved employment prospects. In addition, families expect that by raising their children bilingually, they can maintain the family's heritage language and culture (Bialystok, 2001; King \& Mackey, 2007; Yoshida, 2008). Previous studies of the effects of language experience on bilingual speech production have mostly focused on immigrant bilinguals, namely those who start learning Mandarin after they have immigrated to the Mandarin-speaking environment or the second generation of immigrants who speak the local language and also their parents' Southern Min. Morever, the factors found to be relevant to immigrant bilinguals have also been confirmed in studies of societal bilinguals, namely those who speak two languages that are both required and used in the community or society. (Peng, 1993; Guion et al., 2000a; Guion, 2003).

Since Taiwan's immigration laws were not fully established until 2007, marriage had been the sole source for in-bound population in Taiwan before. According to the statistics of the National Immigration Agency of Taiwan (National Immigration Agency 2018), till the end of 2018, altogether 117,022 new immigrants had moved to Taiwan due to marital relation.

The number of immigrants increases tremendously in Taiwan. Most of them are foreign brides from Southeast Asia, with Vietnamese, Indonesian and Thai as the three largest groups. In the early 1990s, a group of Hakka-Chinese Indonesian women married Taiwanese men and 
migrated to live in Taiwan. These Hakka-Chinese Indonesian women had preferred marrying men of the same ethnicity, owing to the Chinese Exclusion movement in effect since the mid-1960s in Indonesia. Since the mid-1990s, though, about 400,000 Chinese and Southeast Asian women have migrated to Taiwan by virtue of marriage. Moreover, In 1992, the Taiwanese government implemented the Employment Service Act to alleviate Taiwan's labor shortage. This opened the doors to Southeast Asian workers (Chiao, 2008). In 1993, the Taiwanese government's "Go South" policy encouraged investment in the Southeast Asian countries, such as Indonesia,the Philippines and Vietnam (Chen, 1996).

Following studies on Mandarin development by children of Indonesian immigrants (Kuo, 2008, 2009, 2010, 2011) in this study, the researcher investigated of Mandarin, Indonesian, and Taiwanese vocabulary size for children of Indonesian immigrants in Taiwan used Peabody Picture Vocabulary Test for Mandarin. There are four pictures on a page and each pictures's below have a number to assess lexical comprehension in Indonesian and Mandarin. For Taiwanese, the measure tool is an application develepod by Kuo (2017) refferring to PPVT by presenting four pictures to let children point out the target word to measure their vocabulary. Along with a questionnaire, we see the factors affect children's lexical development relationship between mother and children's scores.

\section{Research Questions}

The formulation of the research questions for this study are :

1. What are the vocabulary score in Indonesian, Mandarin and Taiwanese language for children of Indonesian immigrants in Taiwan?

2. What kinds of words do they know in Indonesian, Mandarin and Taiwanese?

3. What factors are related to children's lexical development? 


\section{Significance of study}

This study aim to evaluate the the vocabulary size in Indonesian, Mandarin, and Taiwanese for children of Indonesian immigrants in Taiwan. This study focus on the lexical development. Specifically, this paper seeks to explore the lexical development in Indonesian, Mandarin and Taiwanese acquistion of children of Indonesian immigrants in Taiwan. The result of this study is to raise the awareness and help people to have a better understanding of children of immigrants multilingual lexical development.. 


\section{CHAPTER II}

\section{LITERATURE REVIEW}

\section{Multilingualism}

Located in Southeast Asia, Taiwan is basically composed of four main ethnolinguistic groups: Southern Min, Mainlanders, Hakka, and Austronesian aborigines. Taiwan Southern Min-Mandarin bilinguals typically acquire Southern Min earlier than Mandarin. They learn and use Southern Min as their first language (L1) at home and Mandarin as (L2) the second language at school (Huang and Fon, 2007). This multilingual nation was in due course joined by some other ethnic groups at the turn of this century when new cross-border marriage immigrants, mainly from Mainland China and Southeast Asian countries, arrived. Their arrivals brought with them their native languages, which thus came into contact with the already-existing local languages, widening the linguistic repertoire of Taiwan (Chen,2010).These recent immigrants originate in a variety of ethnicities; some are Han Chinese from Mainland China, others are mainly from Southeast Asian countries, with Vietnamese as the largest group, followed by Indonesians, Thai, and then Filipinos. They brought with them their native languages and cultures (Chen, 2010).

\section{Lexical Development}

There are two major theories of lexical development: learning theory and developmental theories. According to learning theories, children learn the meanings of their first words by associative learning. That is, by repeated exposure to a word (e.g., hearing doggie) paired with a particular experience (e.g., seeing the family dog), the child makes connections between the word and meanings. Exclusively replying on associative learning would be slow and result in many errors. However, children's learning of words is rapid, predictable, and remarkably accurate. Developmental theories, consider semantic development within the wider context of the child's unfolding social, cognitive, and linguistic 
skills (Gleason, 2012).

In addition, there are children in the world who acquire two languages simultaneously in the early stages of language acquisition as a result of their nurture in an environment dealing with more than one language. Much scientific evidence indicates that bilingual children are distinguished by cognitive features that are better than monolingual children where cognitive mechanisms, which are weak to the monolingual, can be enhanced. However, researchers need to conduct a lot of research on the impact of bilingualism on cognitive processes (Kroll $\&$ De Groot, 2005).

Furthermore, studies employing trilingual or multilingual subjects not only offer the opportunity to investigate the acquisition and processing by testees representing the majority of the world's population but also offer new perspectives on the study of language acquisition in general. For instance, a study by Abunawara (1992) showed that the number of connections between the lexicons is higher at lower levels of proficiency. The employment of trilingual versus bilingual participants made very clear that the focus on more than two languages offers invaluable insights not only into multilingual processing but also into psycholinguistic aspects of language learning in general (Herdina\&Jessner, 2002).

The lexicon has always been at the centre of interest in studies on bilingual individuals and/or second language learners. Discussions on the nature of the acquisition of the lexicon have concentrated on questions concerning similarities and differences between lexical operations in L1 and L2 learning and the relationship between form and meaning in processing one or two languages. Many of the studies on bilingual representation and processing focus on the conceptual and lexical or associative links in the bilingual mental lexicon (several studies in Harris 1992 and Schreuder \& Weltens 1993; Singleton 1999.).The lexicon of a language is central to language acquisition as it provides a unique window on the process of acquisition for language as a whole. Currently, the issue of vocabulary acquisition has drawn more and more attention in second language pedagogy and research. In particular, the role of negotiation of meaning in L2 vocabulary acquisition has drawn the attention of many researchers (Pica, 1993, 1994; Long, 1996; Ellis, 1985, 1995, Loschky, 1994; Fuente,2002, 2006; Blake, 2000; Luan \& Sappathy, 2011; Bitchener, 2003; Ellis \& He, 1999; Ellis \& Heimbach, 1997; Ellis, et al., 1994). Meanwhile, vocabulary knowledge constitutes a major aspect of language competence. It is a component of language proficiency. Vocabulary continues to expand throughout the life span (Lenneberg, 1967; Nation, 1990). It grows during infancy, early childhood, school age (age from six through twelve years), adolescent 
years (age from thirteen through eighteen years) and continues to develop even into adulthood (Nation, 1990; Nippold, 1998).Therefore, vocabulary is crucial not only because words allow speakers to express themselves, but also because of the strong association between vocabulary and reading. Very young children understand the pragmatic intent of adults' utterances before they can understand the words themselves. This earlier comprehension is at the emotional, social, and contextual levels. Only very slowly do children come to understand the use of words independent of context (Gleason 2012).

\section{Vocabulary Size}

When vocabulary size is the measure of vocabulary in both languages is taken into account, bilingual toddlers show a total vocabulary size comparable to the same measure in monolingual children. However, in bilinguals, total vocabulary measures may differ from 'conceptual' vocabulary, when translation equivalents (words with the same meaning, usually present from the early stages of lexical development) are counted just once. This is a highly relevant issue to take into account, especially because bilingual toddlers may easily be misidentified as having smaller vocabulary than monolingual toddlers; if the tool used to measure expressive vocabulary is not well adapted to bilingual context's a 'communicative development inventory' should be administered for each of the ambient languages for a correct comparison with monolingual data When vocabulary size is the measure of vocabulary in both languages is taken into account, bilingual toddlers show a total vocabulary size comparable to the same measure in monolingual children. However, in bilinguals, total vocabulary measures may differ from 'conceptual' vocabulary, when translation equivalents (words with the same meaning, usually present from the early stages of lexical development) are counted just once. This is a highly relevant issue to take into account, especially because bilingual toddlers may easily be misidentified as having smaller vocabulary than monolingual toddlers; if the tool used to measure expressive vocabulary is not well adapted to bilingual context's a 'communicative development inventory' should be administered for each of the ambient languages for a correct comparison with monolingual data (Core et al., 2013; Hoff, Core, Place, Rumiche, Señor \& Parra, 2012; Hoff, Rumiche, Burridge, Ribot \& Welsh, 2014)

Other studies have focused on the relationship between vocabulary size in each of the ambient languages and the amount of input received. Results show a positive correlation between these two factors, at least in the early stages, up to the moment when a critical mass 
of lexical items has been reached. It has to be mentioned, however, that beyond direct exposure provided by parents, media, and community, predominant usage of one of the languages may favor a greater than expected vocabulary growth in one of these languages.

\section{Language Input}

Language input refers to "the language to which learners were exposed" (Gass\&Selinker, 2001, p.259). The amount of language input significantly influences children's vocabulary growth (Place\& Hoff, 2011). For vocabulary building, the amount of language input may not always influence language acquisition. The learning of each word needs only a threshold amount of exposure, which is called a "critical mass" (Pearson, 2007,p. 401). That is, once a child figures out the meaning of a new word, then further input may have no effect. But input must provide new words or else children's vocabulary size will not grow, no matter how many old words they hear every day (Pearson, 2007).

Quantity of input in language interactions has been shown to be a strong predictor of young children's vocabulary for monolinguals (Huttenlocher, Haight, Bryk, Seltzer, \& Lyons, 1991). Though they did not measure absolute quantity, Pearson, Fernández, and Oller (1997) found a relative effect of quantity of input on vocabulary in their study of young simultaneous bilinguals; vocabulary knowledge in each language was related to amount of exposure in that language.

Mandarin lexical development for children with Indonesian mothers were found to be slower than children with Taiwanese mothers (Kuo, 2015). Because of that, the researcher attempts further to examine lexical comprehension in Indonesian, Mandarin and Taiwanese language for children of Indonesian immigrants in Taiwan, the second largest non-Mandarinspeaking immigrant group in Taiwan. The researcher investigated whether Indonesian mothers in Taiwan with limited Mandarin proficiency are able to provide the kind of input to facilitate their children's Indonesian, Mandarin and Taiwanese acquisition or not. 


\section{CHAPTER III \\ METHODOLOGY}

The participants consist of 30 Indonesian mothers and their children in Taiwan. The children's age ranges from 7 to 21 they consist of 1 male and 21 female, and Indonesian mother's ages range from 27 to 40. Mandarin and Taiwanese are used in most family. All their fathers are Taiwanese, who are native speakers of Mandarin and Taiwanese. Indonesian mothers usually speak Mandarin to their children. Their length of stay in Taiwan varies from seven to 20 years. Most of Indonesian mother's education level is high school but some mothers have college degree.

\section{Measures}

\section{Indonesian and Mandarin}

The Chinese version of Peabody Picture Vocabulary Test (Lu \& Liou, 1998) was adopted to assess the participants'receptive vocabulary. It was translated from Peabody Picture Vocabulary Test-Revised (Dunn \& Dunn,1997). The Chinese version of Peabody Picture Vocabulary Test, was translated into Indonesian language.(See Appendix1).

PPVT scores serve as estimates of vocabulary growth as a consequence of language intervention (cf. Rice \& Hadley, 1995), as a description of growth in vocabulary in early childhood (cf. Rice, 2009), and as a validity comparison for growth in other indicators of language acquisition, such as the mean length of utterance (Rice, Redmond, \& Hoffman, 2006).

The PPVT measure of vocabulary features multiple choice items in which four pictures are shown for each vocabulary word (including verbs, nouns, and adjectives).The picture from this test is like the following sample in Figure 1. The respondent is instructed to select the picture that best illustrates the definition of the word (read aloud by an examiner, who then scores the response as correct or incorrect). For example, if the experimenter ask a child "who is smiling"? He or she needs to paste a sticker to the picture. The test is given verbally and takes about 20-30 minutes and the test started from their age. Age in circles refers to the lowest age in the 6 month or 12 month phase, for example: The first question is for starting point 3 years 0 months up to 3 years and 5 months, question the 30 th is for 5 years to 5 years and 5 months, the 90th question is for 12 years to 12 years and 11 months. In this test has a 
procedure, every age, if there are eight consecutive correct questions can be continued until the stage exceeds the participant's age limit, but if there are 6 consecutive wrong questions should be stopped.

PPVT-R does not require child verbal responses, it is easy to score, correlates highly with Stanford-Binet IQ scores, has high reliability, and has a very high ceiling. That is, it can be administered to persons from early childhood through older adulthood with norms available for each age group. In order to know the validity of PPVT-III, this study used scores of eight special populations (speech impaired, language delayed, language impaired, mentally retarded [child and adult], reading disabled, hearing impaired, and gifted) with demographically matched control groups (Dunn \& Dunn,1997).

\section{Taiwanese}

The measure tool for Taiwanese is an application develepod by Kuo (2017) refferring from PPVT by presenting four pictures in the an to let children point out the target word to measure their word. It takes only about 20 minutes for each 30 examinee. At the beginning of test, there were three practice trials. There are four pictures on a page and in this test there are 50 picture questions. The picture from this test is like the following sample in Figure 2. The recorder asked a word out of the four pictures and asked the examinees to response the question by pointing to the picture to precede this test. For instance, if the recorder ask "Which one is dog"? Then easily point out the number three picture or say out three. The internal reliablity in test $\alpha=.99$. Southern Min Vocabulary Test Measurement was reliable.

\section{Data Collection}

Data collection proceess in several steps. First, parents answered the questionaire from the researcher about information including level of education for each parent; how long the parents had been in Taiwan; their age (Indonesian mothers and children) ; the language(s) spoken at home during daily life.(See appendix 3) Second, in a week, the children were tested at home first in Indonesian of Peabody Picture Vocabulary. Finally, the last meeting children have test in Mandarin with the the Chinese version of Peabody Picture Vocabulary Test and Taiwanese Picture Vocabulary Test.

In the first week children and Indonesian mothers were tested in Indonesian and Taiwanese, but for mother they need filled the questionnaire (See Apendix 3). One week 
later, they have tested in Mandarin. Each test took about 20 to $40 \mathrm{~min}$. Each child and Indonesian mother's presented with a series of cards, each of which has four pictures, and the child asked to name one of the four pictures that matches the examiner's word spoken in Indonesian. The child and Indonesian mother's needed to point to the picture that matches the spoken word. The next procedure to assess the children's Indonesian and Mandarin and receptive vocabulary in response to items on the PPVT-R Form L (Dunn \& Dunn, 1981). Again, the children were shown four pictures, and the examiner asked the child to point to one picture that matches the spoken Indonesian or Mandarin word. Each child was praised and positively encouraged as much as possible during each test and after the tests. Mothers presented during the child testing but were asked kindly to stay quiet during the tests and not to give the child any hints.

\section{Data Analysis}

Peabody Picture Vocabulary Test have totally one hundred twenty-five and the total points are one hundred twenty-five questions. Where the testing starts from the person being tested. But in this study to measure the Indonesian language does not depending on the age because the Indonesian language is not their first language. The criteria for scoring were based on how many questions participants answer correctly and each question can get one point. The measure tool for Taiwanese language have the total questions are fifty and the total points are one hundred. The criteria for scoring were based on how many questions participants answer correctly and each question can get two points. After the participants took the test, we can get the percentage of accuracy in the vocabulary tests. From the scores we may know the performance of those children and mother. After the scores, all were computed the data were coded and then analysized. Firstly, descriptive statistics such as means, and standard deviations are computed to determine. Secondly, correlation were conducted in order to determine relationship between children's lexical development and their mother's of vocabulary in Indonesian, Mandarin and Taiwanese and other factors. A map of list vocabulary was created to the determine the words do they know in each language. 


\section{CHAPTER IV \\ RESULTS AND DISCUSSION}

\subsection{Vocabulary size in Indonesian, Mandarin and Taiwanese for children of Indonesian immigrants in Taiwan.}

Table 4.1 Descriptive statistic for children's scores.

\begin{tabular}{llll}
\hline & Mean & $\%$ & $S D$ \\
\hline Indonesian score & 25.13 & 20.11 & 7.234 \\
Mandarin score & 38.10 & 74.71 & 12.732 \\
Taiwanese score & 71.00 & 71 & 14.983 \\
\hline Total Score & 134.23 & & 21.757 \\
\hline
\end{tabular}

Table 4.1 showed the raw score and percentage for Indonesian score $(M=25.13)$

Mandarin score $(M=38.10)$ and Taiwanese score $(M=71.00)$. Because of the big difference in scoring standard between PPVT for Indonesian and Mandarin with Taiwanese Vocabulary Test for Taiwanese, Indonesian and Mandarin scores have been converted to 100 based. From the obtained mean is distributed 125 from all PPVT questions and multiplied by 100, then we can get the percentage.This is used to balance results with Taiwanese.

Based on 100 scoring, the mean for Indonesian score is $(M=20.11 \%)$, Mandarin score $(\mathrm{M}=74.71 \%)$ and Taiwanese score $(\mathrm{M}=71.00 \%)$. Mandarin score is better than Taiwanese score, and Indonesian score. According to repeated measures, the difference between the mean is statistically significant score in Indonesian, Mandarin and Taiwanese language, F $(2,58)=(175,955), p=.000$. Three paired score were used to make post hoc comparison 
between Indonesia, Mandarin and Taiwanese. Paired-wise comparisons were showed in Table 4.2.

Table 4.2

Multiple comparison for Indonesian score, Mandarin score and Taiwanese score.

\begin{tabular}{lllll}
\hline Language & (I)score & $(\mathrm{J})$ score & Mean Difference (I-J)
\end{tabular}

$\begin{array}{llll}\text { Indonesian } & \text { Mandarin } & -54.599^{*} & .000 \\ & \text { Taiwanese } & -50.893^{*} & .000 \\ \text { Mandarin } & \text { Indonesian } & 54.599^{*} & .000 \\ & \text { Taiwanese } & 3.706 & .838 \\ & & 50.893^{*} & .000 \\ \text { Taiwanese } & \text { Indonesian } & & .838\end{array}$

Result showed that paired-wise comparisons in Indonesian score is significantly different from Mandarin score and Taiwanese score $(p<0.05)$. The difference between Mandarin and Taiwanese did not reach significance $(p>0.05)$.

\subsection{The words they know each language}

The second question of this study asked "what kinds of words do they know in Indonesian, Mandarin and Taiwanese language "?. In this section presents kinds words they know in each language. In this study the researcher used Peabody Picture Vocabulary Test for Indonesian and Mandarin, and Vocabulary Test for Taiwanese language test. 


\section{INDONESIAN LANGUAGE}

Where the conditions of this measures, depending on the age of the person being tested. But in this study to measure the Indonesian language does not depending on the age because the Indonesian language is not their first language, we started from the first word and ended. Table 4.2 showed the number and percentage of childern that answered each correctly.

Table 4.3. The words that answered correctly by the children in Indonesian language

\begin{tabular}{|c|c|c|c|}
\hline No & Word & Children who answered correctly & \% \\
\hline 1 & Bus & 25 & 83 \\
\hline 2 & Hand & 24 & 80 \\
\hline 3 & Bed & 23 & 76 \\
\hline 4 & Tire & 21 & 70 \\
\hline 5 & Snake & 20 & 66 \\
\hline 6 & Helicopter & 20 & \\
\hline
\end{tabular}




\begin{tabular}{|c|c|c|c|}
\hline 7 & Ship & 19 & 63 \\
\hline 8 & Drum & 19 & 63 \\
\hline 9 & Wardrobe & 18 & 60 \\
\hline 10 & Knee & 17 & 56 \\
\hline 11 & Vegetable & 17 & 56 \\
\hline 12 & Tricycle & 16 & 50 \\
\hline 13 & Bandage & 15 & 50 \\
\hline 14 & & 15 & \\
\hline 15 & Elbow & 15 & \\
\hline & & & \\
\hline
\end{tabular}

As can be seen fom Table 4.2, there are fifteen words answered correctly by the children of this test. The second column show the English translation of words in Indonesian language, the third column show how many children answered the word and the fourth column show the percentage. The percentage shows the proposition of the words that answered correctly among the children's. The words they know include noun categories such as vehicle (bus, helicopter, ship, and tricycle), body part (hand, knee, elbow, and shoulder), furniture (bed, and wardrobe), animal ( snake), instrument (drum) and plant (vegetable). According to this table, we can know the semantic categories they know most are the vehicles and body parts.

\section{MANDARIN LANGUAGE}

The measure used for Mandarin was also Peabody Picture Vocabulary Test. But measure for Mandarin language started from the age of the person being tested. Table 4.3 showed that words that answered correctly by the children and number and of children 
percentage.

Table 4.4. The words that answered correctly by the children in Mandarin language

\begin{tabular}{|c|c|c|c|}
\hline No & Word & Children who answered correctly & $\%$ \\
\hline 1 & Waterfall & 29 & 96 \\
\hline 2 & Kiss & 29 & 96 \\
\hline 3 & Ghost & 29 & 96 \\
\hline 4 & Brood & 29 & 96 \\
\hline 5 & Syringe & 28 & 93 \\
\hline 6 & Bulb & 28 & 93 \\
\hline 7 & Archery & 28 & 93 \\
\hline 8 & Walk & 28 & 93 \\
\hline 9 & Anger & 28 & 93 \\
\hline 10 & Bow & 27 & 90 \\
\hline 11 & Chain & 27 & 90 \\
\hline 12 & Citrus & 26 & 86 \\
\hline 13 & Cornea & 26 & 86 \\
\hline 14 & Entomologist & 26 & 86 \\
\hline
\end{tabular}




\begin{tabular}{|c|c|c|c|}
\hline 15 & Anatomy & 25 & 83 \\
\hline 16 & Carrion & 25 & 83 \\
\hline 17 & Climb & 25 & 83 \\
\hline 18 & Dry & 25 & 83 \\
\hline 19 & Pyramid & 25 & 83 \\
\hline 21 & Inflate & 24 & 80 \\
\hline 22 & Outdoor & 24 & 80 \\
\hline 23 & Verticle & 24 & 80 \\
\hline 24 & Difussion & 24 & 80 \\
\hline 25 & Arrogant & 24 & 80 \\
\hline 26 & Archives & 24 & 80 \\
\hline 27 & Spatula & 24 & 80 \\
\hline 28 & Horoscope & 24 & 80 \\
\hline 29 & Diamond & 24 & 80 \\
\hline 30 & Think & 24 & 80 \\
\hline 31 & Penetrate & 24 & 80 \\
\hline 32 & Joy & 24 & 80 \\
\hline 33 & Serious & 23 & 76 \\
\hline
\end{tabular}




\begin{tabular}{|c|c|c|c|}
\hline 34 & Decorate & 23 & 76 \\
\hline 35 & Bind & 22 & 73 \\
\hline 36 & Dwarf & 22 & 73 \\
\hline
\end{tabular}

As can be seen from Table 4.3, there are thirty - six words that answered correctly by the children of this test. The second column show the English translation of the word in Mandarin language, the third column show how many children answer the word correctly and the fourth column show the percentage of children that answered correctly. The percentage shows the proposition of the words that answered correctly among the children's. The words they know include noun categories such as nature (waterfall), name (ghost, anatomy), articles in home (bulb,), articles in hospital (syringe), articles for daily life (spatula,chain and archives) sports (archery, and bow), part of eyes (cornea), fruit (citrus), human (entomologist), building (pyramid), horoscope, difussion, and shape (diamond). Verb categories include stative verbs (brood, kiss), motion verbs (walk), action verbs (climb and decorate), process verbs (inflate, penetrate, and bind) and cognitive verbs (think). And adjective categories such as human propensity (anger, arrogant, serious and joy),and physical (dwarf, dry). According to this table, we can know the semantic categories they know most are process verbs and human propensity.

\section{TAIWANESE LANGUAGE}

The measure tool for Taiwanese language is an application develepod by Kuo (2017). Table 4.4 showed that words that answered correctly by the children and percentile the number and percentage of children that answered each word correctly.

Table 4.5 The words that answered correctly by the children in Taiwanese language 


\begin{tabular}{|c|c|c|c|}
\hline No & WORD & Children who answered correctly & $\%$ \\
\hline 1 & Fish & 30 & 100 \\
\hline 2 & Dog & 30 & 100 \\
\hline 3 & Milk & 30 & 100 \\
\hline 4 & Hand & 30 & 100 \\
\hline 5 & Mother & 30 & 100 \\
\hline 6 & White & 30 & 100 \\
\hline 7 & Money & 30 & 100 \\
\hline 8 & Flower & 30 & 100 \\
\hline 9 & Train & 30 & 100 \\
\hline 10 & Meat & 30 & 100 \\
\hline 11 & Head & 30 & 100 \\
\hline 12 & Older sister & 30 & 100 \\
\hline 13 & Grand mother & 30 & 100 \\
\hline 14 & Table & 30 & 100 \\
\hline 15 & Car & 29 & 96 \\
\hline 16 & Door & 29 & 96 \\
\hline
\end{tabular}




\begin{tabular}{|c|c|c|c|}
\hline 17 & Pineapple & 29 & 96 \\
\hline 18 & Chair & 29 & 96 \\
\hline 19 & Orange & 29 & 96 \\
\hline 20 & Feet & 29 & 96 \\
\hline 21 & Drink & 29 & 96 \\
\hline 22 & Four & 29 & 96 \\
\hline 23 & Banana & 28 & 93 \\
\hline 24 & Cook & 28 & 93 \\
\hline 25 & Cup & 28 & 93 \\
\hline 26 & Big & 28 & 93 \\
\hline 27 & Snake & 27 & 90 \\
\hline 28 & Fly & 27 & 90 \\
\hline 29 & Thin & 27 & 90 \\
\hline 30 & Corn & 27 & 90 \\
\hline 31 & Frog & 26 & 86 \\
\hline 32 & T-Shirt & 26 & 86 \\
\hline 33 & Red & 26 & 86 \\
\hline
\end{tabular}




\begin{tabular}{|c|c|c|c|}
\hline 34 & Earing & 26 & 86 \\
\hline 35 & Scissors & 26 & 86 \\
\hline 36 & Cut & 25 & 83 \\
\hline 37 & Fierce & 25 & 83 \\
\hline 38 & Carry On & 25 & 83 \\
\hline 39 & Clingy & 25 & 83 \\
\hline 40 & Fishing & 24 & 80 \\
\hline 41 & Same & 24 & 80 \\
\hline 42 & Broken & 24 & 80 \\
\hline 43 & Step Up & 24 & 80 \\
\hline 44 & Scare & 24 & 80 \\
\hline 45 & Ship & 23 & 76 \\
\hline 46 & Wear & 21 & 70 \\
\hline 47 & Shatter & 21 & 70 \\
\hline
\end{tabular}

As can be seen from Table 4.4, there are fourty seven words answered correctly by the children of this test. The second column show the English translation of the word in the word in Taiwanese language, the third column show how many children answer the word and the fourth column show the percentage of the words. The words they know include noun categories such as animal (fish, dog, snake, and froq ), money, colour (white, red), substance (milk), body part ( 
hand, head, and feet), vehicle ( train, car, and ship), food (meat), plant (flower, and corn) kinship ( mother,older sister, and grand mother), furniture (table, chair), number (four), building (door), fruit (pineapple, banana), articles for daily life (cup and scissors), clothes (T-shirt), and accessories (earings 86\%). Verb categories include stative verb (drink, broken, wear and cut), action verb ( carry on, fishing), and motion verb (step up). And adjective categories include physical properties (big, thin), human propensity (fierce, and scare), and value adjectives (the same and clingy). According to this table, we can know the semantic categories they know most are animals.

For Indonesian language, noun dominate most of children's lexicon. Children have smaller receptive vocabulary size for Indonesian than Mandarin and Taiwanese. For Mandarin and Taiwanese, most of children know nouns than verbs, and adjectives. Children have larger receptive vocabulary size in Mandarin and Taiwanese. It seems that the vocabulary growth in the Indonesian language by children of Indonesian immigrants is slower than Mandarin and Taiwanese language. In Figure 1, we can see words of different difficulty in Indonesian, Mandarin and Taiwanese. Children know one word both in Indonesian and Taiwanese. But in Mandarin we can see different words. It is because the measure for Mandarin the children were tested starting with their age in Mandarin. Therefore, in Mandarin we can see the difficult word of children according to their age.Taiwanese words are from high frequency words of 2- 4 years olds. As we can see from Figure 2, there are no overlapping between Indonesian, Mandarin and Taiwanese language. This is because according to the conditions of PPVT, the test started with participant's age. But in this study, measuring the Indonesian language does not begin with participant's age because the Indonesian language is not their first language and for an Indonesian norm is not available. Therefore, for an Indonesian test started from the first item. For Mandarin, they started with participant's age. Consequently, we find domain specific in Indonesian, Mandarin and Taiwanese. This finding showed Mandarin and Taiwanese dominate, because the words they know in Mandarin and Taiwanese are more than Indonesian.

\subsection{Factors related to children's lexical development}

The third question of this study asked "what factors are related to children's lexical development?". In this section showed the raw score and percentage mothers score in each language.. 
Table 4.5 Descriptive statistic for mothers score.

\begin{tabular}{lccc}
\hline & Mean & $\%$ & $S D$ \\
\hline Indonesian score & 96.333 & $77 \%$ & 10.927 \\
Mandarin score & 59.167 & $47 \%$ & 14.071 \\
Taiwanese score & 76.600 & $76 \%$ & 12.952 \\
\hline Total Score & $\mathbf{2 3 2 . 1 0}$ & & $\mathbf{2 5 . 8 6 2}$ \\
\hline
\end{tabular}

Based on 100 scoring, the mean for Indonesian score is $(M=77 \%)$, Mandarin score $(\mathrm{M}=47 \%)$ and Taiwanese score $(\mathrm{M}=76 \%)$ Indonesian score is better than Mandarin score, and Taiwanese score. According to repeated measures, the difference between the mean is statistically significant score in Indonesian, Mandarin and Taiwanese language,

$\mathrm{F}(2.58)=(79.119), p=.000$.

Table 4.6. correlations children score between their's mother

Indonesian score $\quad$ Mandarin score $\quad$ Taiwanese score

Indonesian score

$(r)$

124

318

Mandarin score

(r) 124

125

Taiwanese score

(r) $\quad 318$

125 
MotherIndonesian

score

(r) $\quad 059$

$-196$

$-091$

MotherMandarin

score

(r) $\quad-030$

303

063

MotherTaiwanese score

(r) $\quad 278$

Stay in Taiwan

(r) $\quad 202$

Language at home

(r) 096

175

$373^{*}$

*. Correlation is significant at the 0.05 level (2-tailed).

**. Correlation is significant at the 0.01 level (2-tailed).

According to the Table 4.6, we found that there was positive correlation between children's Taiwanese score and mother's Taiwanese score $(\mathrm{r}=368)$, but for Indonesian score and mother Indonesian score have no correlation. This result could be influenced by the language used at home, because mothers always speak Mandarin or Taiwanese with their children. We can see there was positive correlation between language at home with Taiwanese score for children $(r=373)$. And last correlation we found was correlation between mother's length of stay in Taiwan and Taiwanese score $(r=650)$. Their mother's length of stay in Taiwan have positive correlation with Taiwanese score of children, in this study their mother's length of stay in Taiwan from 10 years until 28 years. This influenced in Taiwanese score, because their mother's are accustomed to using Taiwanese even with vocabulary limitations. 


\section{CHAPTER V \\ CONCLUSION}

\section{Summary of the study}

The goal of the present study was to investigate Mandarin, Indonesian, and Taiwanese vocabulary size for children of Indonesian immigrants in Taiwan. To summarize the present the study, there are three major findings. First, the finding of this study showed that of Indonesian vocabulary score were found to be lower than Mandarin and Taiwanese scores and Mandarin score higher than Taiwanese score. It is because their mother never spoke Indonesian language to their children. Most mothers think that they did not insist that their children speak mothers' native language because (a) mothers did not think that their children had the opportunity to use the language, (b) their children were too young to learn the language, (c) their children did not want to learn the language, (d) learning other languages was more important than learning mothers' native language (Mandarin and Taiwanese were given higher priority than Indonesian), and (e) mothers are worried learning two different languages at the same time may confuse children. Second, this findings showed that the vocabulary ability in each language for children. The words they know in Indonesian are dominated by noun and most of the semantic categories they know is vehicle and body part. For Mandarin and Taiwanses most of children know more nouns, verbs, and adjectives. In Mandarin most of the semantic categories they know are process verbs and human propensity, while in Taiwanese, the semantic categories do they know most is animal. Lastly, the results also showed the factors related to children's lexical development. The result of study show correlattion between Indonesian mothers and her childrens, this study found that there was positive correlation between children's Taiwanese score and mother's Taiwanese score $(\mathrm{r}=368)$, but for Indonesian score and mother Indonesian score have no correlation, because Indonesian mother usually speak Mandarin to their children in daily life. Some mothers spoke Indonesian with their children, but most of them communicate with Mandarin or Taiwanese. 


\section{Limitations and Implications}

There are several limitations of this study that affect the findings and, therefore, they call for careful interpretation of the findings. First, in this study, the researchers used the PPVT-R test for Indonesian and Mandarin. The conditions of this measures, the test started with participant's age. But in this study to measure the Indonesian language does not begin with participant's age because the Indonesian language is not their first language and for an Indonesian norm is not available. Therefore, for the Indonesian test was started from the first item. For Mandarin, they started with the participant's age. Meanwhile, the Taiwanese measures does not have conditions like PPVT-R, this test all the same items are give to all the items. Peabody Pictute Vocabulary Test and Taiwanese Vocabulary Test have the biggest difference scoring standard. PPVT had difficult scoring standard, but Taiwanese Vocabulary Test's scoring standard was easy. Because of that, the comparison of Mandarin and Taiwanese scores has an unbalanced score. Secondly, due to time limitation the data collection only collect participants in Chiayi city and Douliu city. There may be some differences if this test used in other cities of Taiwan. Because different cities may have different factor to influence the result.

In future study, researcher recommends to have more participants and in other regions of Taiwan such as Taipei, Taichung, Tainan etc. The result of this study have a few suggestions for mothers. Indonesian mother's may need to provide special opportunities (such as culture/language instruction on weekends) to support children's Indonesian vocabulary growth so children do not lose proficiency in Indonesian. Having grandparents and other family members visit and speak in Indonesian language with children or inviting children for summer vacations to Indonesia. This research should help people to have a better understanding about the phenomenon of multilingual family, where multilingual has become common in the society. Immigrant children's multilingual experiences are important literacy resources because these experiences help broaden their worldviews and reinforce their language development. 


\section{REFERENCES}

Alice Sterling Honig and Yili Xu. (2012). Chinese Immigrant Families and Bilingualism Among Young Children. NHSA Dialog A Research-to-Practice Journal for the Early Intervention Field. Syracuse University, Department of Child and Family Studies,323G Lyman Hall, Syracuse, NY 13244.

Benedict, H. (1979). Early lexical development: comprehension and production. Journal of Child Language 6, 183-200.

Benson., Janette \& Haith, Marshall M. (2009). Language, Memory, and Cognition in Infancy and Early Childhood, 55-56. Department of Psychology University of Denver, Colorado, USA.

Bitchener, J. (2003). The value of negotiated interaction for learning vocabulary. The 16th educational conference Melbourne, Auckland University of technology.

Blake, R. (2000). Computer mediated communication: A window on L2 Spanish interlanguage. Language Learning and Technology, 4(1), 120-136.

Blackwell, A. A. (2005). Acquiring the English adjective lexicon : relationships with input properties and adjectival semantic typology. Journal of Child Language 32, 535-62.

Bialystok, E., Luk, G., Peets, K. F., \& Yang, S. (2010). Receptive vocabulary differences in monolingual and bilingual children. Bilingualism: Language and Cognition, 13, 525531.

Calfee, R., \& Drum, P. (1986). Research on teaching reading. In M. C. Wittrock (Ed.), Handbook of research on teaching (3rd edition) (pp. 804-849). New York: Macmillan

Cobo-Lewis, A., Pearson, B., Eilers, R., \& Umbel, V. (2002). Effects of bilingualism and bilingual education on oral and written English skills: A multifactor study of standardized test outcomes. In D. K. Oller \& R. Eilers (Eds.), Language and literacy in bilingual children (pp. 64-97). Clevedon, UK: Multilingual Matters.

Core, C., \& Hoff., E. (2013). Input and Language Development in Bilingually Developing Children. Journal Speech Language. Florida Atlantic University, Davie, Florida.

Core, C., Hoff, E., Rumiche, R., \& Señor, M. (2013). Total and conceptual vocabulary in Spanish-English bilinguals from 22 to 30 months: implications for assessment. 
Journal of Speech, Language, and Hearing Research, 56, 1637-1649.

Dixon, L. Q., Zhao, J., Quiroz, B., \& Shin, J. -Y. Home and community factors influencing bilingual children's ethnic language vocabulary development. International Journal of Bilingualism.

Dixon, L. Q. (2011). The role of home and school factors in predicting English vocabulary among bilingual kindergarten children in Singapore. Applied Psycholinguistics, 32, $141-168$.

Dunn, L. M., \& Dunn, D. M. (1997). Peabody picture vocabulary test (3rd ed.). Circle Pines, MN: American Guidance Service.

Duursma, E., Romero-Contreras, S., Szuber, A., Proctor, P., Snow, C. E., August, D., et al. (2007). The role of home literacy and language environment on bilinguals' English and Spanish vocabulary development. Applied Psycholinguistics, 28, 171-190.

Ehab Abunuwara. (1992) .The structure of the trilingual lexicon. European Journal of Cognitive Psychology,4:4, 311-322.

Ellis, R. (1985). Teacher-pupil interaction in second language development. In S. Gass, \& C. Madden (Eds.), Input in second language acquisition (pp. 69-85). Rowley, MA: Newbury House.

Ellis, R., \& Heimbach, R. (1997). Bugs and birds: Children's acquisition of second language vocabulary through interaction. System, 25(2), 247-259

Ellis, Nick C. (2002). Frequency Effects in Language Processing. Studies in Second Language Acquisition, 2(24), 143-188.

Ellis, R., Tanaka, Y., \& Yamazaki, A. (1994). Classroom interaction, comprehension and the acquisition of second language word meanings. Language Learning, 44, 449-491.

Fuente, M. (2002). Negotiation and oral acquisition of L2 vocabulary. SSLA, 24, 81-112.

Fuente, M. (2006). Classroom L2 vocabulary acquisition: Investigating the role of pedagogical tasks and form-focused instruction. Language Teaching Research, $10(3), 263-295$

Gathercole, V. C. M., Thomas, E. M., \& Hughes, E. (2008). Designing a normed receptive vocabulary test for bilingual populations: A model from Welsh. International Journal of Bilingual Education and Bilingualism, 11, 678-720. 
Gleason, J. B. (2012). The development of language (8th ed.). Boston: Pearson.

Hall, C. J. (2002). The automatic cognate form assumption: Evidence for the Parasitic Model of vocabulary development. International Review of Applied Linguistics (IRAL), 40, 69-87.

Harris, R. J. (Ed.) (1992). Cognitive Processing in Bilinguals. Amsterdam: North-Holland.

Hart, B., \& Risley, T. (1992). American parenting of language-learning children: Persisting differences in family-child interactions observed in natural home environments. Developmental Psychology, 28, 1096-1105.

Hart, B., \& Risley, T. (1995). Meaningful differences in the everyday experience of young American children. Baltimore, MD: Brookes.

Herdina \& Ulrike Jessner (2002). A Dynamic Model of Multilingualism: Perspectives of Change in Psycholinguistics, Multilingual Matters. Guillaume Gentil, Modern Language Centre, OISE/University of Toronto

Hsieh, C., \& Wang, C. (2008). Immigrant wives and their cultural influence in Taiwan. Journal of National Taiwan Normal University: Humanities \& Social Sciences, 53, $101-118$.

Huttenlocher, J., Haight, W., Bryk, A., Seltzer, M., \& Lyons, T. (1991). Early vocabulary growth:Relation to language input and gender. Developmental Psychology, 27, 236248. Jusczyk, P. (1997). The discovery of spoken language. Cambridge, MA: MIT Press.

Krashen, Stephen D. (1981). Second Language Acquisition and Second Language Learning Oxford: Pergamon Press Inc.

Kroll, J. F., \& de Groot, A. M. B. (Eds.). (2005). Handbook of bilingualism: Psycholinguistic approaches. New York, NY, US: Oxford University Press.

Kuo, E. C. Y. (1974a). Bilingual pattern of a Chinese immigrant group in the United

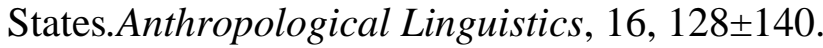

Kuo, E. C. Y. (1974b). The family and bilingual socialization: A sociolinguistic study of a sample of Chinese children in the United States. The Journal of Social Psychology, 92, 181. 